\title{
On the application of ICT in learning English as a second language: IWiLL
}

\author{
C.-H. Kuo ${ }^{1}$, D. Wible ${ }^{2}$, M. C. Chen ${ }^{3}$, H.-Y. Huang ${ }^{4} \&$ S. Kuo ${ }^{5}$ \\ ${ }^{I}$ Department of Computer Science and Information Engineering, \\ Tamkang University, Taiwan \\ ${ }^{2}$ Department of English, Tamkang University, Taiwan \\ ${ }^{3}$ Institute of Information Science, Academia Sinica, Taiwan \\ ${ }^{4}$ Center for Distance Education, Tamkang University, Taiwan \\ ${ }^{5}$ Taipei American School, Taiwan
}

\begin{abstract}
In today's information era, using ICT to enhance teaching and learning is an active field of research. However, the implementation of ICT in teaching and learning is very challenging. In order to build effective teaching and/or learning models, which will be used nationally in Taiwan, the Ministry of Education has initiated a project known as Computer Assisted English Learning: Using ICT in High Schools and Vocational Schools. The IWiLL (Intelligent Web-based interactive Language Learning) system is chosen as the platform to accomplish the above objectives. Two main vehicles were designed, namely, web-based learning activity design and multimedia lecture design, to facilitate the delivery and transaction. In this paper, we describe the design and implementation of these services.
\end{abstract}

Keywords: CALL, ESL, ICT, authoring tool, information retrieval.

\section{Introduction}

Using ICT to enhance teaching and learning is an active research field $[4,11]$. The Ministry of Education in Taiwan has initiated a pilot project that makes use of ICT in high schools and vocational schools to promote the teaching and/or learning strategies and to improve learning performance in English learning. The ultimate goal of the project is to build a nationwide implementation model which 
enhances teachers' IT literacy and to help students improve their learning efficiency.

The deployment of project includes five dimensions as follows: administrative management, IT infrastructure establishment and planning, multimedia lecture design, online learning activity design, and promotion. 12 schools, six high schools and six vocational schools are selected as the core schools to perform the project. In this paper, we describe the portion that makes use of IWiLL, Intelligent Web-Based Interactive Language Learning System as the learning platform $[5,6,10]$ to implement multimedia lecture design and online learning activity design.

The rest of the paper is organized as follows: in Section 2, we will present an overview of this pilot project. Then we present the learning platform, IWiLL in Section 3. The authoring tool that was used to develop multimedia lecture and the lecture management that dealt with management and sharing are shown in Section 4. Online learning activities that were used to pique students' interests in learning, e.g., blog and reading contests, are described in Section 5. Section 6 presents the conclusion and future works.

\section{Overview}

We have witnessed many significant practises by using ICT to improve teaching and learning in class and many industry and commercial institutes. However, it is still a lack of efficient systematic implementation model that can be followed by teacher who lacks of experience and expertise. Organizations may spend a tremendous amount of effort with limited results.

In our approach, the deployment of ICT in high school and vocational school English learning includes five dimensions as follows:

- Administrative management, which focuses on the planning of the school policy to facilitate the implementation.

- IT infrastructure establishment, which sets up computers, multimedia classrooms and network infrastructure.

- Multimedia lecture design, which creates learning content.

- Online learning activity design, which builds a learning model to lift the "classroom boundary".

- Promotion, which addresses the issue of how to spread the established implementation model to the schools' neighbourhoods.

In this paper, we state the portions that make use of IWiLL system in multimedia lecture design and online learning activity design. In the first portion, learning content for students in the form of teacher-guided learning and self-guided learning is created. In the second portion, a learning environment which eliminates the traditional classroom boundary and stimulates learners' interest is established. Integrating the above two-hand strategies, we anticipate that the established teaching and learning models can be promoted nationally and become popular. 


\section{IWiLL system}

IWiLL is a language learning platform. In addition to the basic functionalities of common learning platform, IWiLL conveys many language tools to facilitate content designers creating an interactive language learning sessions. Furthermore, it consists of mechanisms to track learner profiles and lectures sharing. As shown in Figure 1, the toolbar of the authoring tool consists of tools for the operation of the following tasks:

- Configuration of fonts, colors, and type settings.

- Functions of the clipboard, undo/redo, text search and inserting table

- Functions of page control, storing, and checking out

- Functions of inserting hyperlinks, images, essay assignments, movie clips, discussion boards, annotations, TTS, and quizzes.

Most functions in the toolbar are commonly used, so users familiar with any text editing software would be able to use these functions. There are special tools for language learning such as (1) vocabulary-based corpus search, (2) collocation explorer, (3) semantic image retrieval, (4) movie clip search and editing, (5) discussion board, (6) essay assignment, (7) essay writing, (8) assessment, and (9) text to speech (TTS) tools. We present a brief description on collocation explorer, image retrieval system, and mining movies that tools related to create multimedia learning content for English as second language learning.

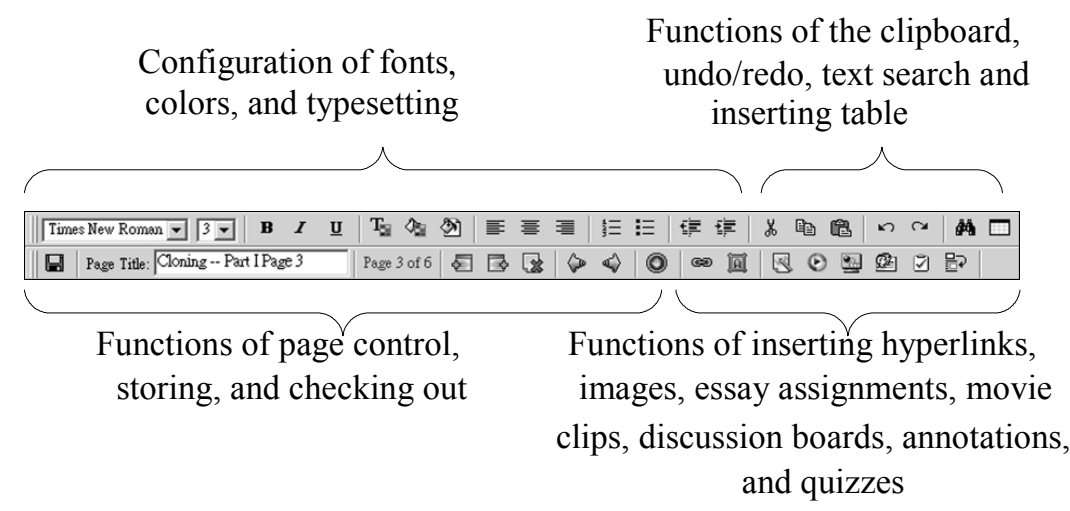

Figure 1: The authoring tool: toolbar.

\subsection{Collocation explorer: Collocator}

Many language resource mining tools in a language learning environment are, in no doubt, very useful. For example, word collocation is one of the most difficult areas for learners in acquiring second language vocabulary. To solve this problem, we have designed a collocation explorer, called Collocator, which is a platform-independent online tool, to address a specific source of this difficulty in any web environment. An apparent source of learning difficulty is that 
collocations are idiosyncratic. For example, make a conclusion is odd whereas draw a conclusion is acceptable and great respect for someone cannot be interchanged with big respect. The tool detects such collocations by exploiting statistical word association measures on a 20-million-word portion of the British National Corpus (BNC) [3]. Combinations of words that achieve a sufficiently high association score in BNC constitute collocations. A link from each detected collocation is added to show additional examples of this same collocation.

\subsection{Images retrieval system}

Images are crucial elements of content [1, 2, 9]. In the image collection and management, we have designed the uploading and annotation subsystems, which allow users to manipulate personal preference images. In the annotation phase, users are requested to describe the uploading images with metadata and main data. The metadata includes category, type (still or moving), texture, color (black and white or color) and so forth. The main data consists of the following items: object (who, what object), event (what action), place (where), time (when), abstract, subject and description. These fields guide the users to provide more complete descriptive features used in semantic indexing and keyword-based search. To provide semantic retrieval features, we integrate keyword extraction, expansion, and filtering in the construction of indices of images. WordNet [12] is used in the keyword expansion mechanism to extend the meanings of keywords and $\mathrm{BNC}$ corpus is used to filter uncommon words.

\subsection{Mining movies}

Video has been presented as an effective medium for computer assisted language learning. One of the most valuable parts of digital video in language learning is the context of the conversation. We transform movies in DVD format to the Internet streaming format, e.g. Microsoft WMV file format. Meanwhile, we extract subtitles of DVD movies to obtain the annotation of movie clips. By using the recently developed DVD technology, we extract the movies' subtitles and the corresponding audio time codes. With the time code of the corresponding subtitles, we are able to synchronize the subtitle texts and movie clips to facilitate queries and broadcasts of movie clips.

In our approach [7], the subtitle text of digital videos collected in the system have been extracted sentence by sentence and have been indexed with their occurring time. To match the application end, we will provide two kinds of query inputs, namely, keyword-based query and syntax-based query, for searching these subtitle texts. Syntax-based query is implemented by a regularexpression-based (regex-based) search engine which is embedded in our system.

Query processing system is in charge of matching the query terms and index terms, then replying to the search result. However, by using regular expression as our query language, this background knowledge of regex would become a bottleneck for our target users. Thus, we have designed a syntax regex query generator shown in Figure 1. This query interface bridges the gap between users and regex, i.e. the users do not have to learn anything about regex. 


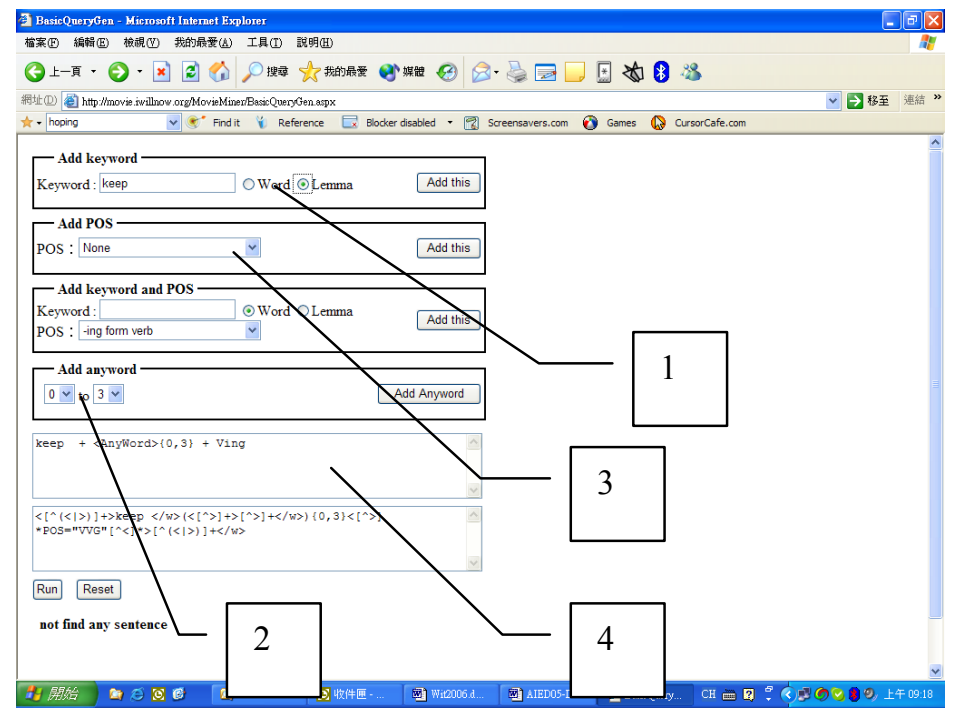

Figure 2: $\quad$ The designed User Interface of Query Generator.

We will use the following example query to show the detailed flow from inputting query to final result representation in this section:

A teacher wants students to learn "the verb keep followed by-ing forms the present participle keeping".

The teacher can use the query generator to produce the regex query, shown in "Regular Expression Query" textbox of Figure 2. The query is composed by simple linear syntax knowledge. As this example, first we input keep as first query term (legend 1 in Figure 2). Since the -ing word can be several words right from keep, we can insert couple words for this elasticity of demand (legend 2 in Figure 2). The last query term, of course, is the part-of-speech "-ing form verb" (legend 3 in Figure 2). The corresponding regex query is generated as legend 4 in Figure 2. From several personal contacts with English teachers in Taiwan, this linear syntax search engine would be beneficial in many English teaching conditions.

The Query Generator then sends the query to the query processor. There are two kinds of data sent to the query processor. One of them is regex query, which would be used in the final step of retrieving process. The other is the information of query terms. These query terms are used to narrow down the search target, which can improve the response time. Using these two query terms, the system can provide a set of candidate clips which subtitle text contain both these two query terms. Although these candidates contain the two query terms, they may not be the final results because they may be very far away from each other in subtitle text. So they still have to be identified by regex query. Figure 3 shows the final results of this example. This figure is the integrated environment of search results and IWiLL authoring environment. 


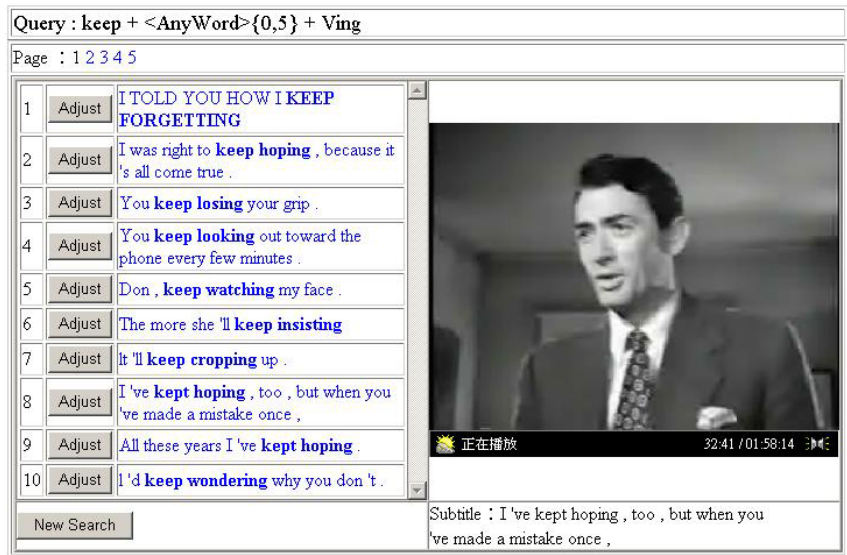

Figure 3: $\quad$ Search results of the corresponding example keep + Ving form.

\section{Multimedia lecture design}

Repeated exposure is an important principle for vocabulary learning [8] but is extremely hard to implement in traditional classroom. Our strategy immerses learners in a digital environment that provides repeated exposure to targeted vocabulary via multiple channels. In this section, we show an example that implements the above popular instructional strategy by combining reading, listening, and writing activities through different media within a learning path by using the developed authoring as shown in Section 3.

\subsection{Lecture design}

Examining the English Taiwan Learner Corpus (TLC) in IWiLL, we found students to have a miscollocation problem in using the phrase acquire knowledge. Due to the way this thought is expressed in Chinese, many students in Taiwan immediately translate the phrase from Chinese into learn knowledge, instead of correctly using acquire knowledge. A lesson called "how to use the word knowledge" is presented in this section to illustrate the content design steps. Note that our main purpose here is to show the power of the authoring tool on the design of learning contents not on the instructional strategy. We would like to include interactive multimedia elements in the lesson as follows.

Reading: sentences to illustrate the meaning of the word "knowledge" and its corresponding usage.

Listening: playback focused movie clips containing target expression in context to deepen learners' impression and understanding.

Writing: writing assignment, sentence writing with image support requiring learner to produce collocation themselves. It is also an evaluation process.

To accomplish the first task, the user makes use of the collocation explorer to get more examples from a 20 million word subcorpus of $\mathrm{BNC}$ [3]. In this case, 
53 sentences are obtained from the IWiLL Collocator, as mentioned in Section 3.1. Partial results are shown in Figure 4. The user selects suitable sentences or modifies these sentences for the lesson content. For the second task, as shown in the previous section, the user may make use of mining movies to obtain and select one or more suitable movie clips in the system. For the third task, the user selects a picture from image database to stimulate learners' creativity and then gives hints to guide learners to write down what they see in the picture using the collocation acquire knowledge in their essay.

\begin{tabular}{|l|l|}
\hline 1 & $\begin{array}{l}\text { However, he cannot always escape the results, or move away; and may not } \\
\text { be able to use this newly acquired knowledge to amend much within the } \\
\text { institution. }\end{array}$ \\
\hline 2 & $\begin{array}{l}\text { This means that a child acquiring language who does not yet know what } \\
\text { chair means but will acquire the knowledge must already have represented } \\
\text { in his "language of thought" a predicate of the kind "is a portable seat for } \\
\text { one". }\end{array}$ \\
\hline 3 & $\begin{array}{l}\text { Some undergraduates seek simply to broaden their horizons and develop } \\
\text { analytical skills; others wish to acquire a more specialized knowledge to be } \\
\text { used in a subsequent career. }\end{array}$ \\
\hline
\end{tabular}

Figure 4: $\quad$ Partial results from IWiLL collocation explorer.

Up to this stage, we have accomplished interactive multimedia learning content design, which includes thinking and learning, and evaluation phases, to learners. The above learning content can be presented in a multimedia classroom or be accessed at many times home at a PC with the Internet access. Then, as learners turn in their essays we enter into an evaluation phase to examine their learning performance.

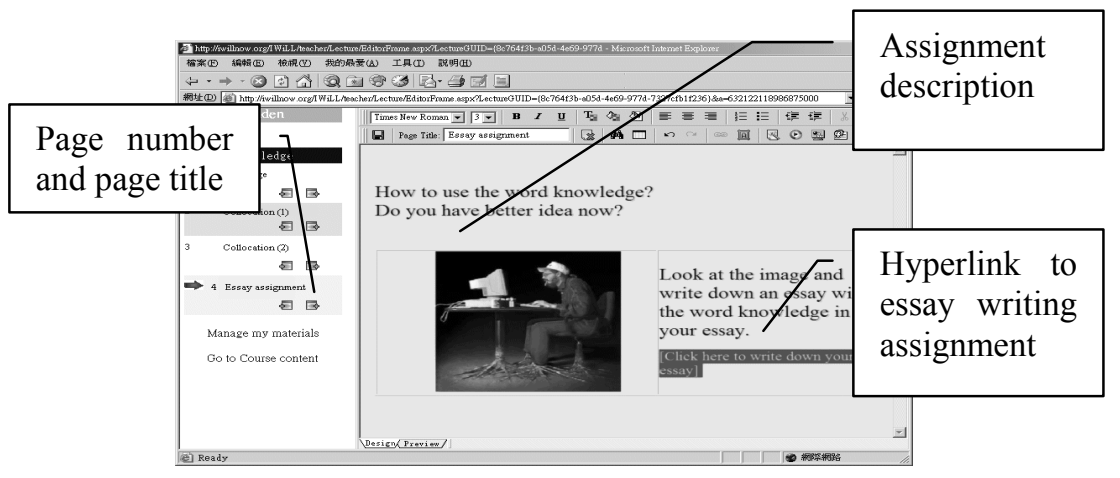

Figure 5: Learning content example: writing assignment.

Our experiences indicate that a content designer is able to create the above interactive multimedia content after one-hour training program in the authoring tool. Therefore, we have better opportunity to explore the power of the web in 
English learning and to build an interactive, informative, and collaborative learning environment.

\subsection{Lecture sharing}

As shown in the above section, a teacher or a content designer is able to create learning content in IWiLL very effectively. Another unique feature that has been implemented in IWiLL is the lecture sharing functionality. Once a lecture is created in IWiLL, the user can decide how to use the lecture, whether it is for the user him/herself, assigned to students, or shared among IWiLL teacher community. The system keeps these sharable contents in a content archive. Teachers are able to browse/search the corresponding database to retrieve the desired lecture content in their teaching. Furthermore, the retrieved content cannot only be assigned to new students or new groups, but also be modified. Meanwhile, a book keep mechanism, which records the number of user contributions and the most popular lecture, is also implemented. Thus, creative and informative lecture content can be reused and shared effectively. As a result, content design in the IWiLL community is not a burden to a teacher, but rather an honor to the content designer.

\section{Online learning activity design}

Online learning activity design serves the purpose of increasing popularity as well as stimulating learners' learning motivation. In order to lift the classroom boundary, these learning activities apply to the intermural scenarios nationwide. We report two learning activities design in this section.

\subsection{Reading club}

We perform online reading contests called the Reading Club twice a year. One is held during winter vacation and the other during summer vacation. Nine different books are selected in each contest. These books are classified into three levels: basic, intermediate, and advanced. Students may select a book or some books to read during vacation. With each book, the Reading Club provides suitable support to assist students in their reading. Meanwhile, we have created discussion board that is hosted by students to engage them in discussions related the book. The creative writing session also encourages learners to express their ideas and thoughts. At the end, a comprehension test is given to evaluate learners' reading achievements. Contests of this type are not novel; however, Reading Club on the Internet has proved to be an effective learning environment which attracts students.

\subsection{Newsletter and blog}

The designed newsletter includes four main subjects: (1) current school reports and news, (2) IWiLL column - Bubba's Monthly Blog, (3) mad about movies, and (4) teacher's lounge. In addition to teacher and students contacts, the 
newsletter is another important way to keep students and teachers informed about events in IWiLL. In the IWiLL column - Bubba's Monthly Blog (see Figure 6), we intend to make use of blog, currently popular among high school and college students, to again stimulate learners' interests. Learners are guided to read an article, then join the blog to enrich individual learning.

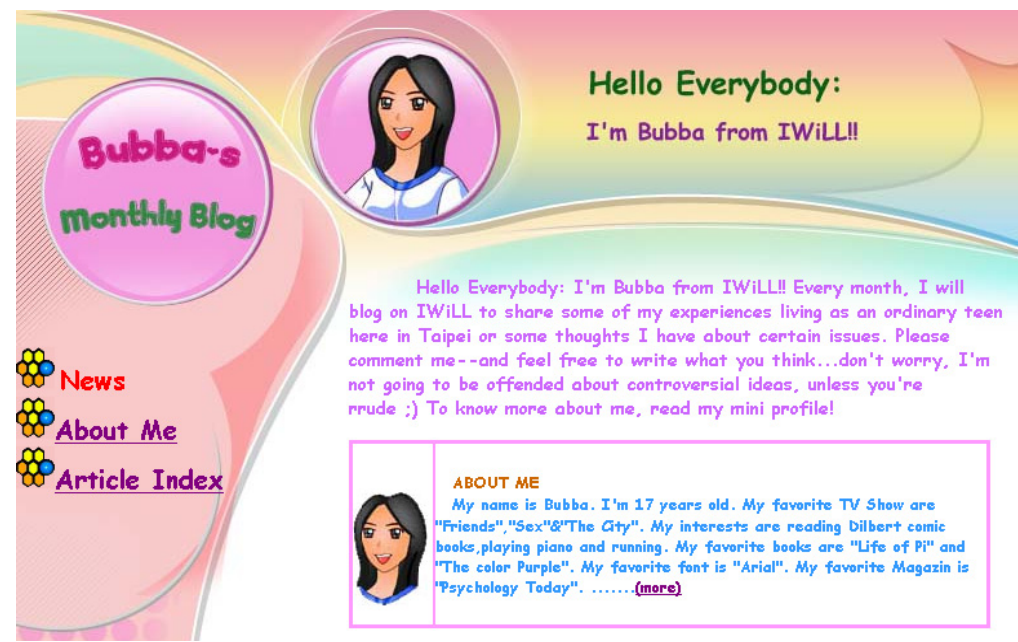

Figure 6: The demo web page of Bubba's monthly blog, http://www. iwillnow.org.

\section{Conclusion}

In this paper, we describe the application of ICT in computer assisted language learning. In the deployment, online learning activity design and multimedia lecture design serve as a two-hand strategy to encourage learners and provide incentive to teachers. A special design web-based authoring tool is equipped with the language learning platform, IWiLL. Content designer or teacher is able to create multimedia lecture content with interactive feature in an easy manner. Without obstacles and unnecessary effort, users can create the virtual learning communities in the IWiLL system. As a result, it not only reduces the lecture preparation loading of teachers but also gains credit to the content designers. Five workshops for introducing this authoring tool have taken place in four different cities in Taiwan, with about 400 participants. So far, we have very encouraging results. In fact, the designed authoring tool can be easily extended to other language learning and other disciplines such as natural science with slight modifications.

Although IWiLL is in its beginning stage, the results have been encouraging so far. There are teachers who organize a team together to create new models by using IWiLL. These types of work are very unusual in the "traditional classroom" and can be implemented only over the Internet with proper setting. 
At present we are pruning the established teaching and/or learning models and online learning activities, which will be applied to high schools and vocational schools systematically and nationwide.

\section{Acknowledgements}

The present work is partly supported by National Science Council under the contract No. NSC (94-2524-S-032-006-) and MoE, Taiwan ROC.

\section{References}

[1] Alessi, S.M., and S.R. Trollip, Multimedia for Learning: Methods and Development, $3^{\text {rd }}$ edition, Allyn \& Bacon, 2001.

[2] Baeza-Yates, Ricardo and Berthier Ribeiro-Neto, "Modern Information Retrieval," ACM Press New York, Addison-Wesley, 1999.

[3] BNC, http://www.natcorp.ox.ac.uk/, 2005.

[4] Horton, W., Designing Web-Based Training: How to teach anyone anything anywhere anytime, John Wiley \&Son, 2000.

[5] IWiLL, http://www.iwillnow.org/, 2005.

[6] Kuo, C., David Wible, and Nai-Lung Tsao, “On Designing a Web-based English Writing Environment and Learner Corpus," IEICE Transactions on Information and Systems, Vol. E84-D, No. 8, August 2001.

[7] Kuo, C., David Wible, Nai-Lung Tsao, and Chen-Fu Chang, "A Video Retrieval System for Computer Assisted Language Learning," AI-ED 2005.

[8] I.S.P. Nation, Learning Vocabulary in Another Language, Cambridge University Press, Cambridge, UK, 2001.

[9] Schank, R., "Active Learning through Multimedia," IEEE Multimedia Magazine, pp.69-78, 1994.

[10] Wible, D., Chin-Hwa Kuo, Feng-yi Chien, Anne Liu, Nai-lung Tsao, and Hong-Sheng Chui, “A Web-based EFL Writing Environment: Exploiting Information for Learners, Teachers, and Researchers," Computers and Education, Vol. 37, pp. 297-315, 2001.

[11] Wible, D., Language Learning and Language Technology - Toward Foundations for Interdisciplinary Collaboration, Crane publishing co., 2005.

[12] WordNet, http://www.cogsci.princeton.edu/ wn/, 2005. 